\title{
Impact of simple, specific, verbal instructions on the quality of bowel preparation in hospitalized patients undergoing colonoscopy: a multicenter randomized controlled trial
}

\section{(ㄷ)(ㅇ) $\Theta$}

Authors

Konstantinos Triantafyllou' ${ }^{1}$, Paraskevas Gkolfakis ${ }^{1}$, Alexandros Skamnelos ${ }^{2}$, Georgia Diamantopoulou ${ }^{3}$, Athanasios Dagas $^{4}$, Georgios Tziatzios ${ }^{1}$, Konstantinos Thomopoulos ${ }^{3}$, Spyros Potamianos ${ }^{4}$, Dimitrios Christodoulou²

Institutions

1 Hepatogastroenterology Unit, Second Department of Internal Medicine - Propaedeutic, Research Institute and Diabetes Center, Medical School, National and Kapodistrian University of Athens, "Attikon” University General Hospital, Athens

2 Division of Gastroenterology, University Hospital \& Faculty of Medicine, School of Health Sciences, University of Ioannina, Ioannina, Greece

3 Division of Gastroenterology, Department of Internal Medicine, University Hospital of Patras, Patras, Greece

4 Department of Gastroenterology, Faculty of Medicine, School of Health Sciences, University of Thessaly, Larisa, Greece

submitted 16.8.2020

accepted after revision $\quad 23.11 .2020$

\section{Bibliography}

Endoscopy International Open 2021; 09: E378-E387

DOI 10.1055/a-1339-0913

ISSN 2364-3722

(c) 2021. The Author(s).

This is an open access article published by Thieme under the terms of the Creative Commons Attribution-NonDerivative-NonCommercial License, permitting copying and reproduction so long as the original work is given appropriate credit. Contents may not be used for commecial purposes, or adapted, remixed, transformed or built upon. (https://creativecommons.org/licenses/by-nc-nd/4.0/)

Georg Thieme Verlag KG, Rüdigerstraße 14,

70469 Stuttgart, Germany

Corresponding author

Paraskevas Gkolfakis, Hepatogastroenterology Unit, Second Department of Internal Medicine, Propaedeutic Research Institute and Diabetes Center, Medical School, National and Kapodistrian University of Athens, "Attikon” University General Hospital, 1 Rimini Street, 12462 Athens, Greece Fax: +30 2105326454

pgkolfakis@med.uoa.gr $\bigoplus$ Supplementary material is available under

https://doi.org/10.1055/a-1339-0913

\section{ABSTRACT}

Background and study aims Bowel preparation for colonoscopy is frequently inadequate in hospitalized patients. We explored the impact of specific verbal instructions on the quality of inpatients bowel preparation and factors associated with preparation failure.

Patients and methods Randomized (1:1), two strata (mobilized vs. bedridden; $3: 2$ ) trial of consecutive inpatients from four tertiary centers, who received either specific, verbal instructions or the standard of care (SOC) ward instructions about bowel preparation. The rate of adequate bowel preparation (Boston Bowel Preparation Score [BBPS] $\geq 6$, no segment $<2$ ) comprised the primary endpoint. Mean BBPS score, good (BBPS score $\geq 7$, no segment score $<2$ ) and excellent (BBPS $=9$ ) were among secondary endpoints. Results We randomized 300 inpatients (180 mobile) aged $71.7 \pm 15.1$ years in the intervention $(49.7 \%$ ) and SOC (50.3\%) groups, respectively. Overall, more patients in the intervention group achieved adequate bowel preparation, but this difference did not reach statistical significance neither in the intention-to-treat [90/149 (60.4\%) vs. 82/151 (54.3\%); $P=0.29$ ] nor in the per-protocol analysis [90/129 (69.8\%) vs. $82 / 132$ (62.1\%); $P=0.19$ ]. Overall BBPS score did not differ statistical significantly in the two groups, but the provision of specific verbal instructions was associated with significant higher rates of good (58.1\% vs. $43.2 \% ; P=0.02)$ and excellent $(31.8 \%$ vs. $16.7 \% ; P=0.004)$ bowel preparation compared to the SOC group. Administration of same-day bowel preparation and patient American Society of Anesthesiologists score $>2$ were identified as risk factors for inadequate bowel preparation.

Conclusions Provision of specific verbal instructions did not increase the rate of adequate bowel preparation in a population of mobilized and bedridden hospitalized patients. 


\section{Introduction}

Adequate bowel preparation is a prerequisite for detection of pathological findings, avoiding repeat colonoscopies and burden lightening both for patients and endoscopy departments [1]. However, hospitalized patients are more prone to inadequate bowel preparation compared to outpatients due to advanced age, comorbidities, physical inactivity, use of medication affecting intestinal motility or non-adherence to regimen's consumption instructions [2]. Use of different purgatives, alterations in the timing of preparation administration, educating patients and/or physicians- nurses are listed among the various interventions that have been implemented aiming to alleviate the effects of this problem. Despite these efforts only two-thirds of inpatients will achieve adequate colon preparation [3], with a recently published European Society of Gastrointestinal Endoscopy (ESGE) guideline recommending specific verbal or written instructions to the patients and to the clinic staff caring for them, to improve the quality of bowel preparation [4]. In this context, we conducted a randomized controlled trial (RCT) aiming to explore the impact of simple, specific verbal instructions on quality of inpatients bowel preparation.

\section{Patients and Methods}

\section{Study design}

A prospective, randomized, single-blinded study that enrolled hospitalized patients who underwent colonoscopy was conducted in tertiary hospitals located in four cities (Athens, Patras, Ioannina, Larissa) in Greece. Ethics committees in all hospitals reviewed and approved the study protocol, as submitted to ClinicalTrials.gov registry (NCT02887014). All patients provided written informed consent at enrollment and the study was performed in compliance with the Helsinki Declaration. Study's design, analysis and interpretation are presented in the CONSORT checklist (Supplementary File A).

\section{Participants}

Inclusion criteria for study enrollment were as follows: (1) any indication for total colonoscopy; (2) age $\geq 18$ years; (3) ability to understand instructions in Greek language; and (4) ability to provide written informed consent. Patients with an indication to undergo sigmoidoscopy, patients with history of colectomy, those without knowledge of Greek language or unable to provide informed consent (e.g. patients with dementia) were not considered eligible for enrollment. The patient's independence level was assessed using the Katz index [5]. To calculate the Katz Index, six different daily living activity domains - bathing, dressing, toileting, transferring, continence, and feeding are assessed. Each domain is assigned a value of 1 or 0 if the activity can be performed independently (no supervision, direction or personal assistance needed) or not (supervision, direction, personal assistance or total care needed) by the patient. A total score of 6 indicates a highly independent individual, whereas a score of 0 indicated a very dependent patient. Based on its component evaluating patient's independence in "transferring" we defined as bedridden any patient not moving at all out of the bed, or requiring a complete transfer from bed to chair. All other patients were considered mobilized.

\section{Instructions development and leaflet administration}

Following a recursive literature search we identified all studies implementing educational interventions to improve inpatients bowel preparation quality [3,6-11]. Based on this evidence, we designed a structured instrument containing the instructions to be verbally disseminated to patients (the study instructions are available in Supplementary File B). Provided information highlighted bowel preparation procedure, the value of adequate bowel preparation for colonoscopy, specific diet during prep, cathartics schedule and the need of drinking the whole volume of the prep and additional clear fluids, as well as, potential side effects (nausea, vomiting, abdominal pain etc.). Specific instructions for the intervention group were kept simple, concise and the time required to deliver them did not exceed 3 minutes. Instructions were pilot tested for comprehension and content among authors and their collaborators.

In detail, patients assigned to the intervention group were visited by one of study's collaborators before the beginning of the preparation who informed them about the aim of the study and then encouraged patients and/or their relatives to carefully read the informed consent leaflet. After signing the consent form, the physician slowly and clearly read a text highlighting that adequate bowel preparation allows the doctor to fully examine the bowel mucosa without presence of any stool residue, that with adequate bowel preparation, easier detection of lesions (e.g. polyps, inflammation) responsible for patient's symptoms is achieved and that adequate bowel cleanliness shortens the examination time and makes it safer for the patient and technically easier for the endoscopist, while it allows the safe and direct excision of lesions (e.g. polyps) detected during colonoscopy. On the contrary, inadequately prepared bowel could impede detection of the problem, lengthen the examination time, tend potential discomfort and lead to repetition of the procedure. The cathartic (polyethylene glycol [PEG]) was provided as powder for oral solution in sachets. Each sachet should be diluted in $1000 \mathrm{~mL}$ of water. The PEG solution should be taken slowly and at a constant rate, approximately one glass $(250 \mathrm{~mL})$ of the solution every 20 to $30 \mathrm{~min}$ utes, with addition of the following liquids: water, tea, juices without fruit residual, sprite, coke and coffee. In case of bloating or nausea, patients could decrease the solution's consumption rate, while ideally, PEG solution consumption was advised to be completed 4 to 6 hours before the time of the scheduled examination.

Patients assigned to the standard of care Group (SOC) (Instructions in Supplementary File B) were similarly visited by one of the study's collaborators before beginning the preparation and informed them about the aim of the study and then encouraged patients and/or their relatives to carefully read the informed consent leaflet. After signing the consent form, no further information was given apart from the SOC information per study center provided by the ward nursing stuff. 


\section{Randomization and concealed allocation}

Inpatients at each medical center with an indication for total colonoscopy were identified the day prior to their examination, during the morning rounds that take place at 8:00 am in each institution. Patients were randomly assigned $(1: 1)$ to receive specific, simple, verbal instructions (intervention arm) or the SOC ward instructions (control arm) by computer-generated randomization with a block design (10 patients per block). Randomization was stratified according to the mobility status of the patients (mobilized vs. bedridden; 3:2). The randomization list was centrally created and sent to one collaborator at each center, who was not actively involved in endoscopies. The sealed envelopes were opened to reveal group allocation once patient consented to participate. Endoscopists were unaware of group allocation, while patients' enrollment among participating centers was done in a competitive way.

\section{Study procedures}

Preparation instructions

Patients randomized to the intervention arm received instructions 12 to 24 hours prior to bowel preparation initiation. A dedicated physician not involved in colonoscopies at each participating center read the instructions at the patient's bedside and/or in front of caregivers without the presence of the ward nursing personnel. At the end of the counseling session, patients/caregivers could raise clarification questions. Moreover, intervention group patients received the standard ward preparation information from the nursing personnel, which was also given to the patients in the $\mathrm{SOC}$ arm.

Patients allocated to the SOC group were informed about colonoscopy prep according to ward routine instructions given by the nursing personnel. These instructions comprised a per center adapted written instruction manual detailing the time points that PEG based regimen should be administered in cases of same-day or split-dose preparation, the volume of water needed to dilute each PEG sachet and finally the need for full adherence to instructions.

\section{Bowel preparation}

Patients received bowel cleansing with $4 \mathrm{~L}$ of PEG solution along with a 2-day low-fiber diet followed by a clear liquid diet the day before the colonoscopy. Bowel preparation was administered either as split doses or as same day (day before) dosing according to time of colonoscopy (morning or afternoon examinations). All examinations were performed 4 to 6 hours after cathartics intake was completed. For the purpose of the study, the total volume of cathartics each patient consumed was also measured. Upon arrival at the Endoscopy Department, each patient was asked to determine how much of the $4 \mathrm{~L}$ preparation they had ingested; each liter represented $25 \%$ of the whole preparation.

\section{Colonoscopy}

Before the start of the colonoscopy, the local study coordinator registered in a secure online database patient satisfaction regarding the colon preparation using a 10-step Likert scale. Thereafter, colonoscopies were performed by endoscopists, who were unaware of the group allocation, using conscious sedation with incremental doses of midazolam and/or pethidine. The local study coordinator using a stopwatch recorded all procedure related times (e.g. cecal intubation and withdrawal times, with pauses for interventions or cleansing). The endoscopist rated the patients' bowel preparation quality using the Boston Bowel preparation scale (BBPS) $[12,13]$. All examiners were previously trained in evaluating BBPS by attending a 10minute lecture and reviewing case videos. When the examination was interrupted, the reason and colon segment where the exam stopped were recorded.

\section{Study endpoints}

Primary endpoint

The impact of the intervention on the number of examinations with adequate bowel preparation, defined by an overall BBPS $\geq 6$ with all segments achieving BBPS $\geq 2$ [13].

\section{Secondary endpoints}

Secondary endpoints were as follows:

- Overall and segmental BBPS score

- Rate of good (overall BBPS $\geq 7$ with all segments achieving BBPS $\geq 2$ ) and excellent (BBPS $=9$ ) bowel preparation

- Identification of risk factors associated with inadequate bowel preparation

- Differences in the cecal intubation rate and examination times (cecal intubation time, withdrawal time and time spent to washout colonic mucosa).

- Patient satisfaction from the bowel preparation

- Side effects related either to the preparation or the examination

- Examination's diagnostic yield defined as the percentage of examinations where their findings justified exam's indication

\section{Statistical analysis and sample size calculation}

Similar studies $[7,8,14]$ in the literature have shown a gain of $20 \%$ in favor of the intervention. Based on 2016 data from the Hepatogastroenterology Unit of Attikon University General Hospital in terms of bowel preparation adequateness (BBPS $\geq 6$, no segment $<2$ ) among inpatients undergoing colonoscopy, a sample size of 300 patients (including a $10 \%$ drop out would be required to detect $18 \%$ improvement for the primary endpoint (from 66\% for inpatients during 2015 to $84 \%$ for outpatients during that period) favoring the intervention group. Statistical significance level $\alpha$ is defined $5 \%$ and the study is powered at the level of $80 \%$. Moreover, randomization of patients was stratified by mobility status anticipating that $40 \%$ of the included subjects would be bedridden based on data of the Athens based center. 
Statistical analysis was performed using the Statistical Package for Social Sciences (IBM SPSS Statistics, Version 25.0. Armonk, New York, United States: IBM Corp). Continuous variables are presented as medians (25th-75th percentile or as mean ( \pm standard deviation; SD) according to visual inspection of the distribution and the Kolmogorov-Smirnov test. Categorical variables are shown as counts (\%) and proportions are presented with the respective $95 \%$ exact binomial Cls. Differences between groups were evaluated using student's t-test and Mann-Whitney U-test in case of continuous data and chi-square or Fisher's exact test in case of categorical data. Regarding adequate colon preparation, we fitted logistic regression models to identify explanatory factors potentially associated with inadequate bowel preparation. Regarding our primary endpoint we used Relative risk (RR) with respective $95 \%$ confidence intervals $(\mathrm{Cl})$ to estimate the effect of the intervention between the two groups. In the univariable models we examined basic demographic characteristics (sex, age, stratum, ASA score and Katz Index), factors potentially affecting the preparation's intake (PEG schema, quantity of PEG ingestion and the person being informed about the significance of an adequate preparation) and factors having been shown in current literature to be related with inadequate bowel preparation (diabetes mellitus, chronic constipation, previous abdominal surgery, previous inadequate colonic preparation, use of tricyclic antidepressants and opioids) $[15,16]$, while in the multivariable model only variables that were associated with the outcome in the univariable models at the level of $\mathrm{P}<0.1$ were included. The Homers-Lemeshow test was used to check the goodness-of-fit of the multivariable regression model. Interaction was tested between each significant factor. Odds ratios (OR) and their 95\% Cls were derived from each variable coefficient in the final model. The significance of each coefficient was tested by the Wald test. Statistical significance was considered for $\mathrm{P} \leq 0.05$. Regarding the primary outcome, we performed intention-to-treat (ITT) and per protocol (PP) analysis excluding incomplete colonoscopies for reason other than bowel preparation inadequateness. Patient's satisfaction, diagnostic yield and adverse event (AE) rates were measured in the ITT population, while we performed PP analysis for the rest endpoints. Finally, to investigate whether the selected stratification model could influence the study's primary endpoint we performed a post-hoc simulation analysis assuming colonoscopy completion and adequateness of bowel preparation rates similar to those detected in our study, but taking into account different theoretical ratios of mobilized to bedridden patients $(60 / 40,70 / 30,80 / 20,90 / 10$, respectively). Once the study database was locked, all authors had access to the data, they reviewed and approved the final manuscript.

\section{Results}

We recruited 300 patients from December 2016 to October 2017. Table 1 lists patient baseline characteristics, examinations-related data, and the contribution of participating centers. Half of the recruited patients received split-dose bowel prep and almost $90 \%$ drank at least $75 \%$ of the cathartic vol-
- Table 1 Patient baseline characteristics and examination-related data.

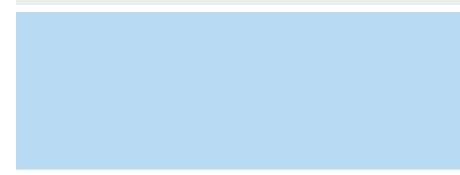

\begin{tabular}{|l|l|}
\multicolumn{2}{|l}{ Allocation group } \\
\hline Intervention & SOC \\
\hline $\mathrm{N}=149$ & $\mathrm{~N}=151$
\end{tabular}

Stratum, n (\%)

- Mobile

- Bedridden

$90(60.4)$

90 (59.6)

- Bedridden

$59(39.6)$

$61(40.4)$

Sex, female (\%)

80 (53.7)

65 (43)

Age, years (mean \pm SD)

$69.9 \pm 16.3$

$73.5 \pm 13.7$

Colonoscopy indication, $\mathrm{n}(\%)$

- Lower gastrointestinal bleeding

55 (36.9)

$76(50.3)$

- Iron deficiency anemia

38 (25.5)

$36(23.8)$

- Abdominal Pain

$10(6.7)$

$5(3.3)$

- Altered bowel habits

$10(6.7)$

$6(4)$

- Metastases of unknown primary

$10(6.7)$

$11(7.3)$

tumor

- Other

$26(17.5)$

$17(11.3)$

Length of stay, days (median, 25th-75th percentile)

Katz Index, n (\%)

\begin{tabular}{|l|l|l|}
\hline$" \leq 3$ & $52(34.9)$ & $52(34.4)$ \\
\hline \hline$>3$ & $97(65.1)$ & $99(65.6)$ \\
\hline
\end{tabular}

ASA Score, $\mathrm{n}(\%)$

- 1

$3(2-6)$

$3(2-5)$

- II

$37(24.8)$

$31(20.6)$

- III

$79(53)$

82 (54.7)

- IV

- IV

$29(19.5)$

34 (22.7)

Diabetes, n (\%)

$4(2.7)$

$3(2)$

Chronic constipation, $\mathrm{n}(\%)$

29 (19.5)

$32(21.2)$

History of abdominal surgery, $\mathrm{n}(\%)$

20 (13.4)

21 (13.9)

History of inadequate bowel prepa-

ration, $\mathrm{n}(\%)$

Tricyclic antidepressants use, $\mathrm{n}(\%)$

$19(12.8)$

25 (16.6)

Opioids use, $\mathrm{n}(\%)$

$7(4.7)$

$5(3.3)$

Preparation administration schedule, $\mathrm{n}(\%)$

\begin{tabular}{|l|r|r|}
\hline - Same day & $75(50.3)$ & $72(47.7)$ \\
\hline - Split dose & $74(49.7)$ & $79(52.3)$ \\
\hline
\end{tabular}

Person informed about bowel preparation, $\mathrm{n}$ (\%)

- Patient

67 (44.9)

- Caregiver

22 (14.8)

25 (16.6)

- Both

60 (40.3)

54 (35.7)

At least $75 \%$ of preparation

ingested, $\mathrm{n}(\%)$ 
- Table 1 (Continuation)

\begin{tabular}{|c|c|c|}
\hline & \multicolumn{2}{|c|}{ Allocation group } \\
\hline & $\begin{array}{l}\text { Intervention } \\
\mathrm{N}=149\end{array}$ & $\begin{array}{l}\text { SOC } \\
N=151\end{array}$ \\
\hline $\begin{array}{l}\text { Incomplete colonoscopies due to } \\
\text { reasons other than inadequate } \\
\text { bowel preparation, } \mathrm{n}(\%)\end{array}$ & $20(13.4)$ & $19(12.6)$ \\
\hline \multicolumn{3}{|l|}{ Reason for incomplete exams, $\mathrm{n}(\%)$} \\
\hline - Obstruction & $9(45)$ & $8(42.1)$ \\
\hline - Intolerance & $9(45)$ & $9(47.4)$ \\
\hline - Technical difficulties & $2(10)$ & $2(10.5)$ \\
\hline $\begin{array}{l}\text { Colonoscopies interrupted due to } \\
\text { inadequate bowel preparation, } \\
\mathrm{n}(\%)\end{array}$ & $6(4)$ & $14(9.3)$ \\
\hline \multicolumn{3}{|l|}{ Center contribution, n (\%) } \\
\hline - $\mathrm{A}$ & $50(33.6)$ & $55(36.4)$ \\
\hline - B & $41(27.5)$ & $36(23.8)$ \\
\hline - $C$ & $32(21.5)$ & $35(23.2)$ \\
\hline - D & $26(17.4)$ & $25(16.6)$ \\
\hline
\end{tabular}

ume. Thirty-nine (13\%) patients were excluded from the PP analysis because colonoscopy was interrupted due to colonic obstruction $(n=17)$, intolerance $(n=18)$ or technical difficulties like acute angulations or redundant colon $(n=4)$. Finally, 20 colonoscopies (6/149 [4\%] and 14/151 [9.3\%]; $P=0.07$ in the intervention and the SOC group, respectively) were interrupted due to inadequate bowel preparation. Thus, 261 inpatients were included in the PP analysis of the primary endpoint (129 and 132 in the intervention and the SOC group, respectively). The study flowchart is presented in $>$ Fig. 1.

\section{Primary endpoint - Rate of examinations with adequate bowel preparation}

In the ITT analysis 90 of 149 patients (60.4\%) in the intervention group and 82 of 151 (54.3\%) in the SOC group $(P=0.29)$ achieved adequate bowel preparation (BBPS $\geq 6$ with no colonic segment receiving $<2$ points). Similarly, no statistically significant difference was detected in the PP analysis [90/129 (69.8\%) vs. 82/132 (62.1\%); $P=0.19$ ].

In the PP analysis of mobile patients, significantly more patients had adequate bowel preparation in the intervention group compared to the SOC group [66/81 (81.5\%) vs. 53/80 (66.3\%); RR(95\%Cl): $1.23(1.02-1.48) ; P=0.03]$, but this statistically significant difference was not detected in the ITT analysis (66/90 [73.3\%] vs. 53/90 [58.9\%]; $P=0.11)$. On the other hand, the intervention had no beneficial impact among bedridden

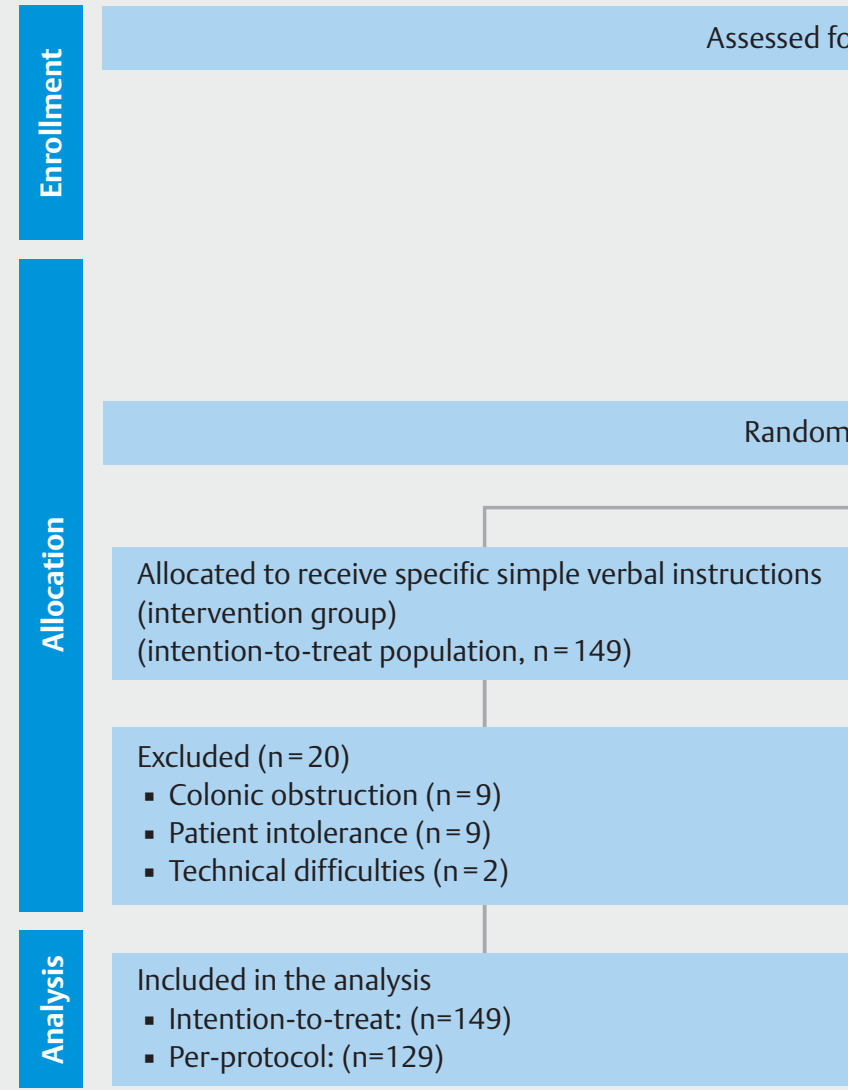

Excluded $(n=44)$

- Not meeting inclusion criteria $(n=32)$

- Indication for sigmoidoscopy $(n=22)$

- History of colectomy $(n=6)$

- No knowledge of Greek language $(n=4)$

- Declined to consent $(n=12)$

Randomized $(n=300)$

Allocated to receive specific simple verbal instructions (intervention group)

(intention-to-treat population, $\mathrm{n}=149$ )

Excluded $(n=20)$

- Colonic obstruction $(n=9)$

- Patient intolerance $(n=9)$

- Technical difficulties $(n=2)$

Included in the analysis

- Per-protocol: $(n=129)$
Allocated to receive standard ward instructions (control group) (intention-to-treat population, $\mathrm{n}=151$ )

Excluded $(n=19)$

- Colonic obstruction $(n=8)$

- Patient intolerance $(n=9)$

- Technical difficulties $(n=2)$

Included in the analysis

- Intention-to-treat: $(n=151)$

- Per-protocol: $(n=132)$

Fig. 1 Study flowchart. 
patients either in the ITT or the PP analysis (24/59 [40.7\%] vs. 29/61 [47.5\%]; $P=0.47$ and $24 / 48$ [50\%] vs. 29/52 [55.8\%]; $P=0.69$, respectively).

\section{Secondary endpoints}

The results of all secondary endpoints are summarized in $>$ Table 2.

\section{Overall and segmental BBPS score changes}

Overall BBPS score did not differ statistically significantly among patients in the intervention and the SOC groups (7 [5$9]$ vs. $6[4.25-8] ; P=0.12)$. However, in terms of segmental BBPS scores, patients receiving the simple, specific verbal instructions achieved better left colon preparation (3 [2-2] vs. 2 [2-3]; $P=0.03$ ) with a concurrent trend toward better colon preparation in the transverse colon $(P=0.08$; $\triangleright$ Table 2$)$.

\section{Examinations with good and excellent bowel preparation}

A significantly higher percentage of subjects that received simple, specific verbal instructions achieved good or excellent bowel preparation compared to patients receiving the standard ward instructions ( $58.1 \%$ vs. $43.2 \% ; P=0.02$ and $31.8 \%$ vs. $16.7 \% ; P=0.004$, respectively).

\section{Predictive factors for adequate bowel preparation}

Using univariable models, four patient-related factors [bedridden status, decreased level of autonomy (Katz index $\leq 3$ ), presence of concurrent morbidities defined as ASA score $>2$ and chronic constipation ( $<3$ bowel movements per weeks for the last 4 weeks)] and two preparation-related factors (same day $4 \mathrm{~L}$ PEG administration and ingestion of less than $75 \%$ of the PEG solution) were associated with inadequate bowel preparation. However, when the multivariable model was applied only same day PEG administration (OR [95\%Cl]: 4.97 [2.41-10.22]) and ASA score > 2 (6.36 [2.67-15.14]) were identified as signif-

\begin{tabular}{|c|c|c|c|}
\hline Outcome & Intervention group & SOC group & $P$ value \\
\hline $\begin{array}{l}\text { Total BBPS } 1 \\
\text { median ( } 25 \text { th-75th percentile) }\end{array}$ & $7(5-9)$ & $6(4.25-8)$ & 0.12 \\
\hline $\begin{array}{l}\text { Right colon BBPS }{ }^{2} \\
\text { median ( } 25 \text { th-75th percentile) }\end{array}$ & $2(1.5-3)$ & $2(1-2)$ & 0.12 \\
\hline $\begin{array}{l}\text { Transverse colon BBPS } 3 \\
\text { median ( } 25 \text { th-75th percentile) }\end{array}$ & $3(2-3)$ & $2(2-3)$ & 0.08 \\
\hline $\begin{array}{l}\text { Left colon BBPS } 3 \\
\text { median ( } 25 \text { th-75th percentile) }\end{array}$ & $3(2-3)$ & $2(2-3)$ & 0.03 \\
\hline $\begin{array}{l}\text { Good bowel preparation }{ }^{1} \\
\text { (total BBPS } \geq 7 \text {, no segment }<2) \\
\mathrm{n}(\%)\end{array}$ & $75(58.1)$ & $57(43.2)$ & 0.02 \\
\hline $\begin{array}{l}\text { Excellent bowel preparation }{ }^{1}(\mathrm{BBPS}=9) \\
\mathrm{n}(\%)\end{array}$ & $41(31.8)$ & $22(16.7)$ & 0.004 \\
\hline $\begin{array}{l}\text { Cecum intubation rate }{ }^{1} \\
\mathrm{n}(\%)\end{array}$ & $123(95.3)$ & $118(89.4)$ & 0.07 \\
\hline $\begin{array}{l}\text { Intubation time (min:sec) }{ }^{1} \\
\text { median ( } 25 \text { th-75th percentile) }\end{array}$ & $10: 00(06: 40-14: 00)$ & $10: 00(07: 45-13: 30)$ & 0.61 \\
\hline $\begin{array}{l}\text { Withdrawal time (min:sec) }{ }^{1} \\
\text { median ( } 25 \text { th-75th percentile) }\end{array}$ & $07: 30(06: 07-10: 00)$ & 07:00 (06:00-08:20) & 0.16 \\
\hline $\begin{array}{l}\text { Washout time (min:sec) }{ }^{1} \\
\text { median ( } 25 \text { th-75th percentile) }\end{array}$ & $02: 00(00: 32-03: 27)$ & 02:00 (01:00-04:07) & 0.26 \\
\hline $\begin{array}{l}\text { Satisfaction }{ }^{3} \\
\text { median ( } 25 \text { th-75th percentile) }\end{array}$ & $8(7-9)$ & $8(6-9)$ & 0.24 \\
\hline $\begin{array}{l}\text { Side effects }{ }^{3} \\
\mathrm{n}(\%)\end{array}$ & $6(4)$ & $3(2)$ & 0.30 \\
\hline Diagnostic yield ${ }^{3}, \mathrm{n}(\%)$ & $84(56.4)$ & $78(51.7)$ & 0.41 \\
\hline \multicolumn{4}{|c|}{$\begin{array}{l}{ }^{1} n=261 \text { (intervention group: } n=129 \text {, control group: } n=132 \text { ); } n=265 \text { (intervention group: } n=132 \text {, control group: } n=133 \text { ); } \\
{ }^{2} n=272 \text { (intervention group: } n=136 \text {, control group: } n=136 \text { ); } n=299 \text { (intervention group: } n=149 \text {, control group: } n=150 \text { ); } \\
{ }^{3} n=300 \text { (intervention group: } n=149 \text {, control group: } n=151 \text { ) higher scores indicate higher satisfaction; } \\
\text { BBPS, Boston Bowel Preparation Score }\end{array}$} \\
\hline
\end{tabular}


- Table 3 Logistic regression analysis of factors associated with inadequate bowel preparation using univariable and multivariable models.

\begin{tabular}{|c|c|c|c|c|}
\hline \multirow[t]{2}{*}{ Variable } & \multicolumn{2}{|l|}{ Univariable model } & \multicolumn{2}{|c|}{ Multivariable model } \\
\hline & OR $(95 \% \mathrm{Cl})$ & $P$ value & OR $(95 \% \mathrm{Cl})$ & $P$ value \\
\hline Sex (female) & $0.65(0.39-1.09)$ & 0.12 & - & - \\
\hline Age $(>75 \text { years })^{1}$ & $1.46(0.87-2.44)$ & 0.19 & - & - \\
\hline Allocation group (intervention) & $0.71(0.43-1.19)$ & 0.24 & - & - \\
\hline Stratum (bedridden) & $2.51(1.48-4.26)$ & 0.001 & $1.55(0.63-3.82)$ & 0.35 \\
\hline PEG administration schema (same day) & $4.14(2.37-7.25)$ & $<0.001$ & $4.97(2.41-10.22)$ & $<0.001$ \\
\hline Ingestion of less than $75 \%$ of PEG solution (no) & $6.73(2.85-15.87)$ & $<0.001$ & $2.23(0.82-6.04)$ & 0.12 \\
\hline Person informed about bowel preparation (patient) & $0.66(0.39-1.12)$ & 0.15 & - & - \\
\hline ASA Score (>2) & $7.19(3.82-13.55)$ & $<0.001$ & $6.36(2.67-15.14)$ & $<0.001$ \\
\hline Katz Index $(1 \leq 3)$ & $1.65(0.96-2.83)$ & 0.09 & $0.83(0.30-2.33)$ & 0.72 \\
\hline Diabetes mellitus (yes) & $1.41(0.75-2.62)$ & 0.33 & - & - \\
\hline Chronic constipation (yes) & $1.98(0.93-4.21)$ & 0.10 & $1.74(0.68-4.45)$ & 0.25 \\
\hline History of abdominal surgery (yes) & $0.69(0.33-1.47)$ & 0.37 & - & - \\
\hline History of inadequate bowel preparation (yes) & $0.72(0.19-2.77)$ & 0.75 & - & - \\
\hline Tricyclic antidepressants use (yes) & $1.06(0.38-2.96)$ & 1 & - & - \\
\hline Opioids use (yes) & $0.66(0.60-0.72)$ & 0.55 & - & - \\
\hline
\end{tabular}

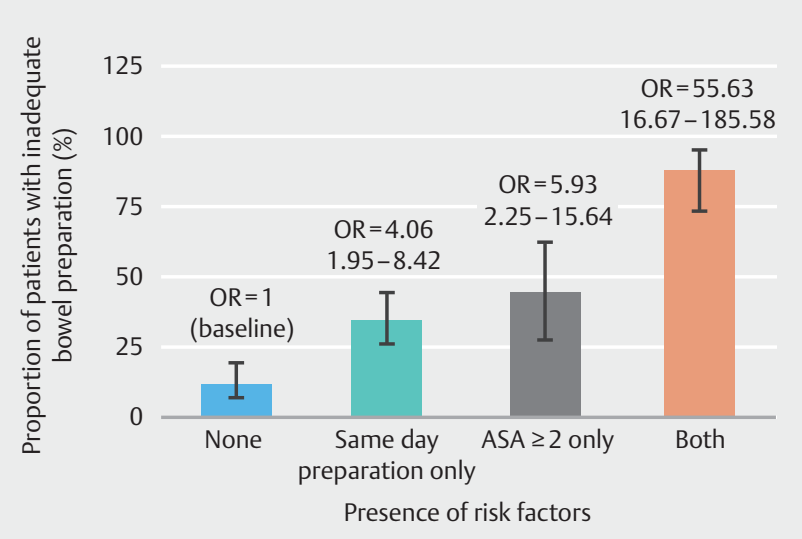

Fig. 2 Proportion of patients with inadequate bowel preparation according to the presence of risk factors. Error bars represent the $95 \% \mathrm{Cl}$ of the proportions. Numbers in parentheses are the $95 \% \mathrm{Cl}$ of the OR.

icant prognostic factors for inadequate bowel preparation ( $\downarrow$ Table 3 ) with the presence of those two factors creating a very strong joint effect that practically invalidates the feasibility of an adequate bowel preparation as may be shown in > Fig. 2 (OR $[95 \% \mathrm{Cl}])$ for the presence of both factors: 55.63 (16.67-185.58).

\section{Cecum intubation rate and examination's times}

We did not detect any statistical significant difference in the cecum intubation rate between the study groups (123/129 or $95.3 \%$ vs. $118 / 132$ or $89.4 \%$; $P=0.07)$. As shown in $>$ Table 2 , neither intubation time, withdrawal time or time spent during the examination to washout the colonic mucosa differed statistical significantly between the two study groups.

\section{Patient satisfaction and side effects}

Using a 10-step Likert scale, the level of patient satisfaction did not differ between the intervention and the SOC groups (8 [79 ] vs. 8 [6-9]; $P=0.24$ ). Overall, nine (3\%) complications occurred in the entire cohort. Of them, six (4\%) in the intervention group and three $(2 \%)$ in the SOC group. More precisely, in the intervention group 3 patients developed AEs related to the bowel preparation itself (nausea while receiving the bowel preparation) and three presented AEs unrelated to the bowel preparation ( 2 patients developed mild pain after the examination and 1 patient was diagnosed with post-polypectomy syndrome, necessitating no further intervention). On the other hand, two and one patient in the SOC group developed nausea during prep ingestion and post-colonoscopy pain, respectively.

\section{Examination diagnostic yield}

Overall, 84 of 149 (56.4\%) and 78151 examinations (51.7\%) in the intervention and the SOC group, respectively, had findings relevant to the examination's indication $(P=0.41)$. Among find- 
ings, diverticular disease and angiodysplasias occurred more frequently.

\section{Post-hoc analysis}

The results of the post-hoc simulation analysis are presented in Supplementary File C. Assuming colonoscopy completion and adequateness of bowel preparation rates similar to those detected in our study, we detected that only in a hypothetical cohort with a 90/10 ratio of mobile to bedridden patients, adequate bowel preparation would have been statistically higher in the intervention group, in both ITT and PP analysis $(P=0.03$ and $P=0.02$, respectively).

\section{Discussion}

Inadequate bowel preparation is a commonly encountered problem among hospitalized patients undergoing colonoscopy. To the best of our knowledge, this is the first multicenter, RCT to show that simple, specific, verbal instructions on top of the SOC failed to significantly improve the quality of bowel preparation among hospitalized patients scheduled to undergo colonoscopy. Of note in our PP analysis, the strata of mobile patients had a significant benefit from the intervention (RR [95\% $\mathrm{Cl}]: 1.23$ [1.02-1.48]). Moreover, we showed that inpatients receiving instructions achieved significantly higher rates of good and excellent bowel preparation. During the study, low recruiting rates of bedridden inpatients were a challenge for all participating centers and we cannot exclude that the initially anticipated $40 \%$ of bedridden inpatients may not sufficiently reflect real life. Noteworthy, when assuming similar rates of colonoscopy completion and adequateness of bowel preparation as those detected in the current study, a hypothetical 90/10 ratio of mobile/bedridden patients would have led to a statistically significant higher rate of adequate bowel preparation, as illustrated in the simulation analysis.

Beyond any doubt, preparing inpatients for colonoscopy is a challenging task, with considerable consequences both for the patients (missing diagnosis, repeat procedures, increased AEs, longer hospitalization) and the healthcare systems, as well [17]. A recent meta-analysis summarizing all methods available to improve colon preparation quality in the inpatient setting, underlined the superiority of educational interventions (specifically designed booklets, written instructions, training either the patients, the healthcare professionals or both) compared to other strategies in improving the odds for adequate bowel preparation [3]. As far as individual RCTs are considered, two studies investigating the effect of educational interventions on hospitalized patients' bowel preparation quality have been published, so far [7, 18]. The former showed that use of an educational booklet improved the odds of an adequate bowel preparation (OR [95\% CI]: 3.14 [1.29-7.83]) [7], while in the latter provision of instructions through a medical smartphone application, complementary to standard oral and written instructions significantly improved the rate of adequate bowel preparation (77.2\% vs. $56.8 \%$ ) [18]. However, both studies -beyond being conducted in a single-center setting- have limitations that merit attention; unknown compliance, protocol violation (twenty randomized inpatients not evaluated because of scheduling conflicts) in one [7], while in the other the content of oral information given to patients may have varied to some extent [18].

Our results are in line with the above-mentioned studies confirming the challenge of adequate bowel preparation (172) 261 or $65.9 \%$ ) in hospitalized patients. Aside from addressing limitations of previous studies, we went a step further and evaluated a simple and inexpensive intervention that could be easily incorporated in everyday clinical practice. A unique finding of current study was that administration of instructions has been beneficial for mobilized patients, leading to more examinations with adequate bowel preparation, compared to those bedridden. Moreover, the impact of the intervention was more favorable in achieving, higher than adequate (good and excellent) bowel preparation. Apparently, both these findings are directly related to compliance, which can be rather low among debilitated patients suffering from major comorbidities, given the complexity of steps involved in successful bowel preparation [19]. In this sense, the rationale of education seems extremely valuable for mobilized patients, who perhaps should be the principal target for healthcare practices. A recent RCT showed that mobilized outpatients receiving written or written and oral instructions achieved high rates of compliance with the preparation schema (split-dose) and consequently adequate bowel cleansing [20].

On the other hand, bedridden status might either prevent successful ingestion of bowel prep or affect instructions comprehension and compliance; thus, bowel preparation in this subset of patients should be handled with multiple, combined strategies on a case-by-case basis [21].

Our multivariable model identified same-day PEG regimen administration (OR [95\%Cl]) 4.97 [2.41-10.22]) and ASA class $>2$ (6.36 [2.67-15.14]) as factors independently associated with inadequacy of colon cleansing. Moreover, the co-presence of those two factors practically precludes the possibility of adequate bowel preparation (OR [95\%Cl]): 55.63 [16.67-185.58]). Contrariwise, split-dose regimens have been recently acknowledged as independent factor significantly reducing the odds of inadequate colon cleansing in a prospective, large, multicenter, observational study among hospitalized patients [16].

Higher ASA score usually correlates with chronically ill patients prone to multiple factors i.e. comorbid conditions (diabetes mellitus, renal disease, chronic obstructive pulmonary disease, chronic constipation, congestive heart failure) that limit tolerability or efficacy of bowel preparation. Moreover, higher ASA class, related to decrease of mobility and physical level of activity, have been consistently identified as predictors of inadequate bowel preparation $[15,19]$. In line with our results, Fuccio et al. [16] - in the most recent prospective, multicenter inpatient study - identified several setting-, patient- and preparation-related factors associated with an adequate bowel preparation in hospitalized patients. Ingestion of at least $75 \%$ of the prep and split-dose regimen were related to increased odds of adequate bowel preparation. Other favorable prognostic factors were the admission to gastroenterology units, the provision of written and oral instructions to patients and the 
administration of $1 \mathrm{~L}$ polyethylene glycol-based bowel purge. On the other hand, bedridden patients, patients with comorbidities (diabetes mellitus), prolonged ( $>7$ days) hospital stay, constipation and intake of anti-psychotic drugs were considered more prone to an inadequate bowel preparation. Although the use of predictive models for inadequate bowel preparation is not yet recommended in clinical practice [4], researchers validated a model to identify patients with inadequate preparation and developed an easy-to-use app to assist clinicians.

Strengths of our RCT include its multicenter design, simulation of real life regarding split-dose or same day-prep administration according to local practice, patient stratification according to mobility status and measurement of patient compliance which was previously unknown (administration of a booklet or a mobile application cannot ensure that patients read or followed what was instructed [7, 18]) Finally, our intervention demands neither specific knowledge nor any additional cost and, as shown by the high rate of patient satisfaction, was very acceptable to patients.

Our study is not without limitations. First, although all the hospitals were tertiary and one could argue about generalizability of the results to non-academic practice settings, the intervention's simplicity favors the opposite. Second, during the study period, a low-volume preparation was not available. Third, we did not investigate the duration of the intervention's effect in the long term and the need for active intervention of nursing personnel for intake of the bowel preparation among bedridden patients was not quantified. Fourth, we excluded patients who could not provide informed consent. One might consider this group as one of the highest risk for non-adherence that may eventually have benefited the most from the intervention. Finally, another limitation is the distinctive roles of bowel preparation adherence vs. pharmacology and their potential association with the study design, itself. Our study was not designed to address these issues and our intervention could only improve patient adherence to preparation.

\section{Conclusion}

To conclude, this multicenter randomized study failed to demonstrate that use of simple, specific, verbal instructions on top of the SOC can improve the quality of bowel preparation in a setting of hospitalized patients undergoing colonoscopy. Despite the fact that a beneficial effect was shown among mobile patients and same-day schedule prep and presence of comorbidities were detected as risk factors for colon preparation failure, additional strategies should be evaluated in future inpatient studies to optimize bowel preparation in this particular subgroup of patients.

\section{Acknowledgements}

This study was presented as an oral presentation during the ESGE Days 2019, Prague, Czech Republic. The study protocol is available at the ClinicalTrials.gov registry, trial number: NCT02887014

\section{Competing interests}

The authors declare that they have no conflict of interest.

\section{References}

[1] Kaminski MF, Thomas-Gibson S, Bugajski M et al. Performance measures for lower gastrointestinal endoscopy: a European Society of Gastrointestinal Endoscopy (ESGE) Quality Improvement Initiative. Endoscopy 2017; 49: 378-397

[2] Mahmood S, Farooqui SM, Madhoun MF. Predictors of inadequate bowel preparation for colonoscopy: a systematic review and meta-analysis. Europ J Gastroenterol Hepatol 2018; 30: 819-826

[3] Gkolfakis P, Tziatzios G, Papanikolaou IS et al. Strategies to improve inpatients' quality of bowel preparation for colonoscopy: a systematic review and meta-analysis. Gastroenterol Res Pract 2019; 2019: 5147208

[4] Hassan C, East J, Radaelli F et al. Bowel preparation for colonoscopy: European Society of Gastrointestinal Endoscopy (ESGE) Guideline Update 2019. Endoscopy 2019; 51: 775-794

[5] Katz S, Downs TD, Cash HR et al. Progress in development of the index of ADL. The Gerontologist 1970; 10: 20-30

[6] Chorev N, Chadad B, Segal N et al. Preparation for colonoscopy in hospitalized patients. Dig Dis Sci 2007; 52: 835-839

[7] Ergen WF, Pasricha T, Hubbard FJ et al. Providing hospitalized patients with an educational booklet increases the quality of colonoscopy bowel preparation. Clin Gastroenterol Hepatol 2016; 14: 858-864

[8] Lee Y], Kim ES, Park KS et al. Education for ward nurses influences the quality of inpatient's bowel preparation for colonoscopy. Medicine (Baltimore) 2015; 94: e1423

[9] Rosenfeld G, Krygier D, Enns RA et al. The impact of patient education on the quality of inpatient bowel preparation for colonoscopy. Can J Gastroenterol 2010; 24: 543-546

[10] Shah-Khan SM, Cumberledge J, Reynolds G]. Using the plan-do-studyact approach to improve inpatient colonoscopy preparation. BM] Open Qual 2017; 6: e000230

[11] Chambers K, Whiteman K, Stephens K et al. Improving inpatient colonoscopy preparation in a university hospital: an evidence-based practice project. Gastroenterol Nurs 2016; 39: 86-94

[12] Lai E], Calderwood AH, Doros $G$ et al. The Boston bowel preparation scale: a valid and reliable instrument for colonoscopy-oriented research. Gastrointest Endosc 2009; 69: 620-625

[13] Calderwood AH, Schroy PC 3rd et al. Boston Bowel Preparation Scale scores provide a standardized definition of adequate for describing bowel cleanliness. Gastrointest Endosc 2014; 80: 269-276

[14] Guo X, Yang Z, Zhao L et al. Enhanced instructions improve the quality of bowel preparation for colonoscopy: a meta-analysis of randomized controlled trials. Gastrointest Endosc 2017; 85: 90-97 e96

[15] Dik VK, Moons LM, Huyuk M et al. Predicting inadequate bowel preparation for colonoscopy in participants receiving split-dose bowel preparation: development and validation of a prediction score. Gastrointest Endosc 2015; 81: 665-672

[16] Fuccio L, Frazzoni L, Spada C et al. Factors that affect adequacy of colon cleansing for colonoscopy in hospitalized patients. Clin Gastroenterol Hepatol 2021: doi:10.1016/j.cgh.2020.02.055

[17] Garber A, Sarvepalli S, Burke CA et al. Modifiable Factors associated with quality of bowel preparation among hospitalized patients undergoing colonoscopy. J Hospital Med 2019; 14: 278-283

[18] Guo B, Zuo X, Li Z et al. Improving the quality of bowel preparation through an app for inpatients undergoing colonoscopy: A randomized controlled trial. J Adv Nursing 2020; 76: 1037-1045 
[19] Yadlapati R, Johnston ER, Gregory DL et al. Predictors of inadequate inpatient colonoscopy preparation and its association with hospital length of stay and costs. Dig Dis Sci 2015; 60: 3482-3490

[20] Andrealli A, Paggi S, Amato A et al. Educational strategies for colonoscopy bowel prep overcome barriers against split-dosing: A ran- domized controlled trial. United Europ Gastroenterol J 2018; 6: 283289

[21] Argyropoulos SK, Mahmood SK, Campbell E] et al. Improving the quality of inpatient bowel preparation for colonoscopies. Digest Dis Sci 2018; 63: 338-344 